BULL. AUSTRAL. MATH. SOC.

$20 \mathrm{M} 20,20 \mathrm{M} 30$

VOL. 35 (1987) $373-378$

\title{
THE MINIMAL FA.ITHFUL DEGREE OF A
}

\author{
FUNDAMENTAL INVERSE SEMIGROUP
}

\author{
D. EASDOWN
}

This paper shows that the smallest size of a set for which a finite fundamental inverse semigroup can be faithfully represented by partial transformations of that set is the number of join irreducible elements of its semilattice of idempotents.

\section{Introduction}

In faithfully representing a given semigroup by partial transformations of a set, it is natural to ask what is the least size of a set for which this is possible. This question, among others, was posed by Schein in [7, Problem 45].

Here, the problem is solved for finite fundamental inverse semigroups. The special case of a semilattice is considered, from which the general case follows by applying a theorem of Munn, which describes fundamental inverse semigroups in terms of principal ideal isomorphisms of semilattices.

\section{Preliminaries}

Standard terminology and basic results relating to inverse semigroups, and semilattices in particular, as given by Howie in [1], will be

Received 19 May 1986.

Copyright Clearance Centre, Inc. Serial-fee code: 0004-9729/87 $\$ \mathrm{~A} 2.00+0.00$. 
assumed. The following result, due to Munn $[3,4]$, will be used in Section 4. Recall that if $E$ is a semilattice, the Munn semigroup $T_{E}$ consists of all isomorphisms between principal ideals of $E$.

THEOREM 1 [1, 4.10]. An inverse semigroup $S$ with semilatice of idempotents $E$ is fundomental if and only if it is isomorphic to a full inverse subsemigroup of $T_{E}$.

Hereafter all semigroups will be assumed to be finite. Define the minimal faithful degree $\mu(S)$ of a semigroup $S$ to be the least nonnegative integer $n$ such that $S$ can be embedded in $P T_{X}$, the semigroup of partial transformations of a set $X$, where $X$ contains $n$ elements. Note that if $T$ is a subsemigroup of $S$ then $\mu(T) \leq \mu(S)$. If $X$ is a set, let ${ }^{i d_{X}}$ denote the identity mapping on $X$.

The following result follows from more general results about inverse semigroups (see for example [5, IV.5.9], [2, II .8.4],[8] or [6]), though a proof is included for completeness.

PROPOSITION 2. Let $E$ be a semilattice and $\phi: E \rightarrow P T_{X} a$ faithful representation. Then there is a faithful representation $\bar{\phi}: E \rightarrow P_{X}$ such that, for aZl $e \in E$,

$$
e \bar{\phi}=i d_{Y} \quad \text { for some } \quad Y \subseteq X \text {. }
$$

Proof. Define $\bar{\phi}: E \rightarrow \mathrm{PT}_{X}$ by, for $e \in E$,

$$
e \vec{\phi}=e \phi \mid \text { range }(e \phi) .
$$

It is routine to verify that $\bar{\phi}$ is a faithful representation. Moreover, if $x \in \operatorname{domain}(e \bar{\phi})$ then $x \in$ range $(e \phi)$, so, for some $y \in X, x=y(e \phi)$, which yields $x(e \bar{\phi})=[y(e \phi)](e \bar{\phi})=[y(e \phi)](e \phi)=y(e \phi)=x$, that is, $e \bar{\phi}=i d$ domain $(e \bar{\phi})$. This completes the proof.

If $E$ is a semilattice, the symbols $\wedge$ and $\vee$ will be used to denote infimum and supremum respectively, when they exist, with respect to the partial order of $E$. Thus $\wedge E$ is the least element of $E$, which exists since $E$ is finite, which will be denoted by 0 , the zero of $E$. Note also that $0=v \varnothing$. Call an element $e$ of $E$ join 
irreducible if $e \neq 0$ and, for $f, g \in E$,

$$
e=f \vee g \text { implies } e=f \text { or } e=g \text {. }
$$

Note that by this definition 0 is not join irreducible. Note also that, since $E$ is finite, any non-zero element of $E$ can be expressed as the join of a set of join irreducible elements. If $e \in E$, define $\bar{e}=\{f \in E \mid f \leq e$ and $f$ is join irreducible $\}$.

LEMMA 3. If $E$ is a semizattice then, for $e \in E, e=v \bar{e}$.

Proof. Let $e \in E$. If $e=0$ then $\bar{e}=\varnothing$ and indeed $e=\sqrt{e}$. If $e \neq 0$ then write $e$ as the join of join irreducible elements $e_{1}, \ldots, e_{n}:$

$$
e=v\left\{e_{1}, \ldots, e_{n}\right\} \text {. }
$$

Certainly $e$ is an upper bound for $\bar{e}$. Suppose also $f$ is an upper bound for $\bar{e}$, so in particular $f$ is an upper bound for $\left\{e_{1}, \ldots, e_{n}\right\}$. Hence $e \leq f$, which shows $e$ is the least upper bound for $\bar{e}$, which completes the proof.

LEMMA 4. Let $E$ be the semilattice which is the subsemigroup of PT ${ }_{X}$ consisting of all partial transformations of a set $X$ which are identity moppings on their respective domains. Then, in $E$, for $Y, Z \subseteq X$,

(i) $i d_{Y} \leq i d_{Z}$ if and only if $Y \subseteq Z$;

(ii) $i d_{Y \cap Z}=i d_{Y} \wedge i d_{Z}$;

(iii) $i d_{Y \cup Z}=i d_{Y} \vee i d_{Z}$.

Proof. These follow immediately from the fact that $i d_{Y} i d_{Z}=i d_{Y \cap Z}$.

LEMMA 5. Let $(P, \leq)$ be cony non-empty finite partially ordered set with $n$ elements. Then there is a listing $P=\left\{p_{1}, \ldots, p_{n}\right\}$ such that, whenever $1 \leq i<j \leq n$,

$$
p_{j} \neq p_{i}
$$

Proof. Define the listing inductively. Let $p_{1}$ be any minimal element of $P$, which exists by finiteness. Assume $p_{1}, \ldots, p_{k}$ have 
been chosen, where $k<n$. Choose $p_{k+1}$ to be any minimal element of $P \backslash\left\{p_{1}, \ldots, p_{k}\right\}$. The minimality of $p_{k+1}$ ensures that the condition of the lemma holds.

\section{Semilattices.}

THEOREM 6. Let $E$ be a finite semilatice with $n$ join irreducible elements. Then $\mu(E)=n$.

Proof. If $E=\{0\}$ then $n=0$ and $\mu(E)=0$, so the statement of the theorem holds. Suppose then $E$ contains a non-zero element, so $n \geq 1$.

Let $X$ be the set of all join irreducible elements of $E$. Define a mapping $\phi: E \rightarrow P T_{X}$ by, for $e \in E$,

$$
e \phi=i d \bar{e} \text {. }
$$

For $e, f \in E, \overline{e f}=\bar{e} \cap \bar{f}$, so

$$
i d_{\overline{e f}}=i d_{\bar{e} \bar{f}}=i d_{\bar{e}} i d_{\bar{f}} \text {. }
$$

which shows $\phi$ is homomorphic. Also, if $\bar{e}=\bar{f}$ then, by Lemma 3 , $e=v \vec{e}=v \bar{f}=f$, so $\phi$ is one-one. Hence $\phi$ is a faithful representation, so $\mu(E) \leq n$.

Suppose now $\psi: E \rightarrow P T_{Y}$ is a faithful representation where $Y$ is a set with $m$ elements. It will be shown that $m \geq n$. Suppose to the contrary that $m<n$.

By Proposition 2, it may be assumed that for each $e \in E, e \psi$ is the identity mapping on some subset of $Y$. Let $X=\left\{x_{1}, \ldots, x_{n}\right\}$, so that for $i=1$ to $n$ there is a subset $X_{i}$ of $Y$ for which $x_{i} \psi=i d_{X_{i}}$.

By Lemma 5 , it may further be assumed that $\left\{x_{1}, \ldots, x_{n}\right\}$ has been listed so that for $1 \leq i<j \leq n, x_{j} \$ x_{i}$, so by Lemma $4, x_{j} \ddagger x_{i}$. Put $y_{i}=X_{1} \cup \ldots \cup X_{i}$, for $i=1$ to $n$. Thus $y_{1} \subseteq \ldots \subseteq y_{n}$ so $\left|y_{1}\right| \leq \ldots \leq\left|y_{n}\right| \leq m<n$.

If $n=1$ then $y_{1}=\varnothing$, so $x_{1} \psi=i d_{\emptyset}=0 \psi$, which contradicts the fact that $\psi$ is faithful. Hence $n>1$, and thus $Y_{k+1}=Y_{k}$ for some 
$k$, where $1 \leq k \leq n-1$, which means that $X_{k+1} \subseteq X_{1} \cup \ldots \cup X_{k}$.

$$
\text { put } X_{i}^{\prime}=X_{i} \cap X_{k+1} \text { for } i=1 \text { to } k \text {, so }
$$

$$
\begin{aligned}
x_{k+1} & =x_{k+1} \cap\left(x_{1} \cup \ldots \cup x_{k}\right) \\
& =x_{1}^{\prime} \cup \ldots \cup x_{k}^{\prime} .
\end{aligned}
$$

Thus, by Iemma 4 , and since $\psi$ is a faithful representation,

$$
\begin{aligned}
x_{k+1} \psi=i d_{X_{k+1}} & =i d_{X_{1}^{\prime}} \vee \ldots \vee i d_{X_{k}^{\prime}} \\
& =\left(i d_{X_{1}} i d_{X_{k+1}}\right) \vee \ldots \vee\left(i d_{X_{k}} i d_{X_{k+1}}\right) \\
& =\left[\left(x_{1} x_{k+1}\right) \vee \ldots \vee\left(x_{k} x_{k+1}\right)\right] \psi,
\end{aligned}
$$

so

$$
x_{k+1}=\left(x_{1} x_{k+1}\right) \vee \ldots \vee\left(x_{k} x_{k+1}\right)
$$

But $x_{k+1}$ is join irreducible, so $x_{k+1}=x_{j} x_{k+1}$, for some $j$ where $1 \leq j \leq k$. Thus $x_{k+1} \leq x_{j}$, so

$$
i d_{X_{k+1}}=x_{k+1} \psi \leq x_{j} \psi=i d_{X}
$$

By Lemma 4, $x_{k+1} \subseteq X_{j}$, which contradicts the fact that $x_{k+1} \notin X_{j}$. This shows $m \geq n$, so $\mu(E) \geq n$.

Hence $\mu(S)=n$, which completes the proof.

4. Fundamental inverse semigroups.

THEOREM 7. Let $S$ be a finite fimdomental inverse semigroup with semilattice of idempotents $E$. Then

$$
\mu(S)=\mu\left(T_{E}\right)=n
$$

where $n$ is the number of join irreducible elements of $E$.

Proof. Clearly $\mu(E) \leq \mu(S) \leq \mu\left(T_{E}\right)$. By Theorem 6, it remains to prove $\mu\left(T_{E}\right) \leq n$.

Let $X=\left\{x_{1}, \ldots, x_{n}\right\}$ be the set of join irreducible elements of $E$. 


$$
\begin{aligned}
\text { Define } \phi: T_{E} \rightarrow P T_{X} \text { by, for } \alpha \in T_{E}, \\
\alpha \phi: x_{i} \rightarrow \begin{cases}x_{i} \alpha \text { if } x_{i} \in \operatorname{domain}(\alpha) \\
\text { undefined otherwise. }\end{cases}
\end{aligned}
$$

Note that an element of a principal ideal of $E$ is join irreducible in that ideal if and only if it is join irreducible in $E$. Hence $\phi$ is well-defined and homomorphic because join irreducible elements are sent to join irreducible elements by semilattice isomorphisms, and one-one because a semilattice isomorphism of a finite semilattice is completely determined by its action on join irreducible elements.

Hence $\phi$ is a faithful representation, so $\mu\left(T_{E}\right) \leq n$, which completes the proof.

\section{References}

[1] J. M. Howie, An introduction to semigroup theory. (London Mathematical Society Monographs 7, Academic Press, London, 1976).

[2] E. S. Ljapin, Semigroups. (Translations of Mathematical Monographs, Volume 3, Amexican Mathematical Society, Providence, R.I., 1974).

[3] W. D. Munn, "Uniform semilattices and bisimple inverse semigroups", Quart. J. Math. Oxford Ser. (2) 17 (1966), 151-159.

[4] W. D. Munn, "Fundamental inverse semigroups", Quart. J. Math. Oxford Ser. (2) 21 (1970), 152-170.

[5] Mario Petrich, Inverse semigroups. (Pure and Applied Mathematics, John Wiley \& Sons, 1984).

[6] G. B. Preston, "Representations of inverse semigroups by one-to-one partial transformations of a set", Semigroup Forwn 6 (1973), 240-245; addendurn : ibid 8 (1974), 277.

[7] L. N. Shevrin, "The Sverdlovsk Tetrad", Semigroup Fomu 4 (1972), 274-280.

[8] V. V. Vagner, "The theory of generalized heaps and generalized groups", Mat. Sb. 32 (1953), 545-632 (Russian).

Department of Mathematics

University of Western Australia

Nedlands, W.A. 6009 